appears either as evidence of a permanent state of emergency or an accurate reflection of the domestic dimension of the »war on terror «. On the other hand, to the extent that preparation for terrorism and tornados falls into the same category, the political mobilization quality of the »war on terror « may actually be diminished by its practical homology with the more politically neutral planning for natural disasters. ${ }^{23}$ Today we find the new security frontier at the intersection of the new paradigm of preparedness and the older national security state.

The politics of security in the United States five years after September 11th is a mixture of institutional adaptation to the new logics of the preparedness paradigm and the old game of political manipulation for partisan gain. Both of these dynamics affect the role of the United States in global security. Preparedness could be a vehicle for latter-day isolationists, or it could be a basis for new forms of international cooperation on

23 Which is not to say that the preparations and consequences of natural disasters cannot be terribly politicized. One need only think of the way Tokyo Mayor Shintaro Ishihara has manipulated public sentiment against Koreans and Chinese during the annual disaster preparedness event on the anniversary of the 1923 Great Kanto Earthquake, or the pathetically botched response to the destruction of New Orleans by Hurricane Katrina in 2005. Nonetheless, it is axiomatic that one cannot attribute intention to a hurricane or earthquake the way one can to a human entity. topics from trade to disaster response. The misuse of power by the Bush Administration could finally call forth a principled response from Congress, or it could result in an even deeper crisis of the rule of law with perilous consequences both at home and abroad.

In the months after the attack there was a gap between those for whom 9/11 »changed everything « and those for whom 9/11 did not warrant a historical caesura. With our very limited hindsight we can nonetheless identify September 11th as a critical event for the politics of security in the United States in that it acted as a catalyst for widespread bureaucratic reorganization (such as the DHS), provided opportunities for audacious policy adventures (as in Iraq), hardened ideologies (as with radical Islamic groups), and propelled the concentration of executive power (as with Bush's championing of the unitary executive). A historical institutionalist may well consider these last years of the Bush Administration a critical juncture, the path dependent outcome of which will determine the security of us all for a long time to come. We can only work to insure that the dynamics set in motion by the attack will be consonant with the rule of law, not its further attenuation. And I still miss the towers every day.

\title{
Der »Dschihadismus«: Gewaltideologie einer politischen Sekte
}

\section{Kai Hirschmann*}

\begin{abstract}
The fight against terrorism with traditional instruments of power like armed forces, police and repressive diplomacy often misses the intended effect. The recognition that organisations of fighters under the ideology of the »jihad « have similarities with the structures of sects, must be taken into account in the fight against the Islamic terror. The leading heads of the Islamic terror are rather "propagandists of the jihad « than hierarchic leaders and, therefore, quickly replaceable. If individual people of a terrorist organisation get killed or force is used on its adherents, the idea of jihad will rather win than lose popularity. Because of that, a theological and political analysis of the ideology of the Islamic terror, by the western-Christian communities and the muslim parishes, must become the aim of all efforts.
\end{abstract}

Keywords: Islamistischer Terrorismus, Terrorismus, Dschihadismus, Sekte, Islamismus, Terrorismusbekämpfung

$\mathrm{D}$ er Kampf ${ }^{1}$ gegen den Terrorismus wird heute weltweit geführt. Leider oft mit untauglichen Mitteln, weil es häufig an einer detaillierten Analyse dieses Gewaltphänomens mangelt. Eine personen- und gruppenbezogene Bekämpfung mit traditionellen Machtinstrumenten wie Streitkräften, Polizei und repressiver Diplomatie läuft häufig ins Leere, weil der Gegner sich nicht in klassischen, hierarchischen oder personenbezogenen Strukturen organisiert hat. Die derzeitigen Protagonisten des weltweiten gewaltbereiten Islamismus und Dschihadismus sind Dienstleister für eine

\footnotetext{
* Dr. Kai Hirschmann, Stv. Direktor, Institut für Terrorismusforschung und Sicherheitspolitik (IFTUS) und Lehrbeauftragter am Institut für Politische Wissenschaft und Soziologie der Universität Bonn.
}

Gewaltideologie, nicht aber deren Schöpfer. Die Organisation von Kämpfern unter der Ideologie des »Dschihad«, die sehr wenig mit dem Islam zu tun hat, ist vergleichbar mit Sekten und ihren Strukturen. Daher muss bei der Bekämpfung des islamistischen Terrorismus bedacht werden: Es handelt sich um eine Idee bzw. Weltanschauung in den Köpfen, die sich weltweit wie eine Sekte strukturiert und verhält.

\section{Charakteristika von Sekten}

Der gewaltbereite Islamismus und Dschihadismus ist bisher selten unter dem Sektenaspekt betrachtet worden, da bei der 
Auseinandersetzung mit dem Phänomen auf die Erfahrungen mit den traditionellen Terrorgruppen der Vergangenheit zurückgegriffen wird. Das neue ideologiegesteuerte Gewaltphänomen ist aber weder an bestimmte Länder und Regionen noch an Personen gebunden, sondern funktioniert über die Anwendung, Interpretation und Weiterentwicklung eines Gewaltkonzeptes, das wesentlich älter ist als Personen wie z.B. Osama bin Laden.

Überwiegend sind es bisher Aufsehen erregende Einzelereignisse, die das öffentliche Bewusstsein zum Thema »Sekten und Gewalt« prägen. Einige Beispiele ${ }^{1}$ :

- Der Massensuizid der Anhänger des »Volkstempels«. Die Aufforderung des Sektenführers Jim Jones: »Die Zeit ist gekommen, uns an einem anderen Ort wiederzutreffen «, führte im November 1978 im Urwald von Guayana zum Tod von 912 Mitgliedern des »Volkstempels«. Unter den Opfern befanden sich 276 Kinder. Sie tranken mit Zyankali versetzte Limonade oder wurden erschossen.

- Das Ende der »Davidianer« in Waco (USA) am 19. April 1993. Dort belagerten Einheiten der Bundespolizei 1993 das Hauptquartier der »Davidianer-Sekte«, einer bis an die Zähne bewaffneten rechtsfaschistisch-apokalyptischen Gruppe, weil diese in ihrer abgeschlossenen Kommune auch ein größeres Waffenlager angelegt hatten. Einer Durchsuchung entzog sich die fundamentalistische Sekte durch Kampf. Als die Gebäude vor laufenden Fernsehkameras aus aller Welt in Flammen aufgingen, verbrannten auch Sektenführer David Koresh und 75 seiner Anhänger.

- Der Selbstmord bzw. Mord der Gruppe der »Sonnentempler« von Luc Jouret und Joseph di Mambro (Schweiz, Kanada, Frankreich 1994/95). Der von Luc Jouret empfohlene »Transit zum Sirius « endete 1994 in Kanada und der Schweiz mit dem Tod von 53 »Sonnentemplern«. In der Folgezeit nahmen sich weitere 21 Mitglieder das Leben.

- Der Anschlag (mit dem chemischen Kampfstoff Sarin) am 20. März 1995 auf das Tokioter U-Bahnnetz durch die Sekte »Aum Shinrikyo« (»Höchste Wahrheit«), angeleitet von ihrem spirituellen Führer Shoko Asahara. Die austretenden Sarin-Dämpfe verbreiteten sich in 15 U-Bahn-Stationen. Durch den Anschlag starben zwölf Menschen, über 5.500 wurden verletzt.

- Der Selbstmord von Mitgliedern der »Heaven's Gate«-Sekte. Um mit einem UFO im Schatten des Kometen Hale-Bopp ins Jenseits zu starten, empfahl Sektenführer M.H. Applewhite im März 1997 seinen Anhängern in den USA, vergifteten Pudding zu essen. Er und 38 Sektenmitglieder starben.

- Im August 1998 bereiteten sich im Südschwarzwald rund 700 Mitglieder der Gemeinschaft »Fiat Lux« auf den bevorstehenden Weltuntergang vor. Am 9. August 1998 sollte der drei Monate andauernde Dritte Weltkrieg beginnen. Danach, so die Prophetin Erika Bertschinger-Eicke alias Uriella, würden sich die Polkappen der Erde verschieben, ein Komet in die Nordsee rauschen, Vulkane in der Eifel ausbrechen und zwei Drittel der Menschheit ertrinken. Das verbleibende

1 Hirschmann/Tophoven/Dietl (2006), Kap. 7; Rohmann (1998), S. 7 und Senatsverwaltung Berlin (1997), S. 5.
Drittel der Menschheit würde daraufhin von Raumschiffen aufgenommen und gerettet werden.

Die Bespiele zeigen, dass auch für absurde Ideen und Konzepte größere Zahlen von Anhängern zu gewinnen sind, die für ihre Überzeugungen (mit Ausnahme von »Fiat Lux«) bereit sind, sich und andere Menschen zu töten. Derartige Sektenstrukturen hat es nicht nur im weltanschaulichen Bereich, sondern auch unter den Anhängern von Buchreligionen immer wieder gegeben. Besonders deutlich wird dies im Bereich christlicher Sekten, ${ }^{2}$ die in ihrer Radikalität teilweise auch vor Gewalt nicht zurückschreckten.

Sprachlich kommt der Begriff »Sekte« von »sequi « (lateinisch »folgen«) und ist die Übersetzung von »hairesis « (griechisch »Wahl, Gefolgschaft«), was diejenigen bezeichnete, die den Anschauungen eines Philosophen folgten. Andererseits wird er abgeleitet von »secare« (lateinisch »trennen, abschneiden «) und bezeichnet eine Gruppe, die sich mit und wegen einer besonderen Lehrmeinung von einer herrschenden Religion gelöst hat. ${ }^{3}$ Nach Gasper/Müller/Valentin wird der Sektenbegriff »als eine Bezeichnung für sich abschließende, in Lehre und/oder Praxis von der Mehrheit abweichend orientierte, somit dissidierende Minderheiten verwendet $«{ }^{4}$ Beckers/Reimer beschreiben derartige »Gemeinschaften« als »eine Abspaltung von einer vorgegebenen Kirche oder Religionsgemeinschaft bzw. eine Sonderbildung in ihrem Raum und mit Bezug zu ihr - , die >sektiererische Züge aufweist $«{ }^{5}$

In der soziologischen und sozialwissenschaftlichen Literatur wird eine »Sekte « bestimmt durch das Maß, in dem sie in Spannung, Widerspruch und Gegensatz zu ihrer Umwelt steht. ${ }^{6}$ Als »Sekte« wird hier eine kleine, exklusive, religiöse oder weltanschauliche, wissenschaftliche oder auch politische Gruppe verstanden, die von ihren Anhängern ein totales Engagement fordert und die dabei ihre Trennung von der Umwelt und deren Zurückweisung besonders betont. ${ }^{7}$ »Sekte « wird mithin als Bezeichnung für sich abschließende, in Lehre und/oder Praxis von der Mehrheit abweichende Minderheiten verwendet. ${ }^{8}$ Sekten versprechen, sowohl die meisten persönlichen Wünsche eines Menschen zu erfüllen als auch eine ganze Reihe von gesellschaftlichen Defiziten auszugleichen. Im Allgemeinen bieten Sektenführer einfache Lösungen für die immer komplexer werdenden Probleme der Welt an. ${ }^{9}$ Im umgangssprachlichen Gebrauch ist der Ausdruck »Sekte« zum Synonym für eine abgeschlossene, der Umwelt gegenüber feindlich eingestellte ideologische Gemeinschaft mit tendenziell konflikthafter bis gefährlicher Lehre und Binnenstruktur geworden. ${ }^{10}$ Folgende Charakteristika und Gemeinsamkeiten solcher Bewegungen (Gruppen) können festgestellt werden: ${ }^{11}$

\footnotetext{
2 Siehe hierzu detailliert Reller/Kießig/Tschoerner (1978).

3 Sprachliche Herleitung nach Wikipedia - die freie Enzyklopädie, Definition "Sekte «.

Vgl. Gasper/Müller/Valentin (1990), S. 973.

5 Vgl. Beckers/Kohle (1994), S. 68.

6 Siehe z.B. Wilson (1970), Johnson (1971) und Stark/Bainbridge (1985); Zusammenfassung bei Enquete-Kommission (1998), S. 19.

7 Vgl. Abercrombie/Hill/Turner (1994), S. 371 und Enquete-Kommission (1998), S. 19 ..

8 Rohmann (1998), S. 6.

9 Zimbardo (1997).

10 Vgl. BMFS (2003), S. 9

11 Vgl. Canadian Security Intelligence Service (2000), S. 2.
} 
- Extremistische oder apokalyptische Überzeugungen.

- Dualismus: Der Glaube, dass die Welt in »Gut« und »Böse« aufgeteilt ist, wobei man selbst das »Gute« repräsentiert.

- Verfolgte Auserwählte: Man sieht sich selbst als auserwählte Elite (Propheten), die von verrückten und tyrannischen Mächten verfolgt werden.

- Determinismus: Die Bewegungen glauben, nur sie können der ultimative Gewinner des »letzten Kampfes« sein. Dabei erscheint eine gewaltsame Auseinandersetzung immer notwendiger.

- Rettung durch Konflikt: Eine (Seelen-)Rettung hängt von der Teilnahme am Kampf ab, dessen Ziel die Vernichtung des Gegners ist.

- Mitgliederkontrolle/totalitäre Strukturen: Die Bewegung (Gruppe) kontrolliert jeden Lebensbereich ihrer Mitglieder. Sie ist totalitär strukturiert.

- Fehlende Hemmschwellen: Da die gesellschaftlichen Normen und Regeln »des Feindes Werk « sind, können sie beliebig missachtet werden.

Die Gründe für den Beitritt zu einer Sekte liegen in der Regel in der Persönlichkeitsstruktur sowie dem Dissens zwischen persönlichen Erwartungen und der Lebensrealität begründet. Sie setzen sich z.B. zusammen aus: ${ }^{12}$

- dem Bedürfnis nach Gemeinschaft, Solidarität und Gruppenzugehörigkeit,

- der Suche nach Lebenssinn, nach Lösungen und nach einer sinnvollen Tätigkeit,

- dem Bedürfnis nach Spiritualität, Wahrheit und religiöser Erfahrung,

- der Sehnsucht nach Orientierung, Richtungsweisung und Zukunftsperspektiven,

- der Suche nach Autorität und geistiger Lenkung,

- dem Vorliegen von Krisen- und Übergangssituationen bzw. kritischer Lebensereignisse, für die der Gegner bzw. die Umwelt verantwortlich gemacht wird,

- der Entwicklung starker negativer Emotionalität gegenüber der politischen, religiösen und/oder gesellschaftlichen Umgebung z.B. durch Verlusterlebnisse.

Im Grunde sind die Argumentationsmuster aller religiösen oder pseudo-religiösen Sekten weltweit vergleichbar, nur die jeweiligen Ideologien und Thesen austauschbar. Die schablonenhafte Weltsicht und die Schlussfolgerungen daraus erscheinen in der Struktur nicht weit voneinander entfernt, da alle Sekten und Glaubensgemeinschaften einen radikal oppositionellen Gesellschaftsentwurf haben, sich von Feinden umzingelt sehen und diese meinen bekämpfen zu müssen. Dabei wähnen sie sich immer im Besitz der einzigen Wahrheit bzw. göttlichen Erleuchtung. ${ }^{13}$ Der Frankfurter Pfarrer Fritz Huth kommt zu dem Ergebnis: »Die Grundstrukturen in christlichen und islamischen Fundamentalistenkreisen sind ähnlich.

12 Auflistung unter Berücksichtigung der Forschungsergebnisse von Rohmann (1998) und der dort in Kapitel 2 aufgeführten Quellen.

13 Hirschmann/Tophoven/Dietl (2006).
Opposition gegen die moderne westliche Gesellschaft, Sehnsucht nach den alten und einfachen Regeln der Schrift. «14

Auch ihre Rekrutierungsmuster sind vergleichbar: Junge Menschen, die in der Gesellschaft, in der sie leben, nicht ganz angekommen sind oder sich ausgegrenzt fühlen, vielleicht sogar brutal unterdrückt werden. Sie werden mit einem Lebens- und Gesellschaftsentwurf konfrontiert, der scheinbar eine Lösung ihrer Befindlichkeit verspricht, ihnen das Gefühl der Fremde nimmt und sie einen Lebenssinn finden lässt. Sind sie einmal in die entsprechende Parallelwelt eingetreten, greift ein Automatismus, der sie immer weiter entfremdet und in der Sekte aufgehen lässt, bis sie bereit sind, die Ziele der Sekte vorbehaltlos zu übernehmen und sich dafür (mitunter mit ihrem Leben) einzusetzen. Negative Erfahrungen mit den vermeintlichen »Feinden « tragen weiter zu einer extremen Emotionalisierung und Radikalisierung bei. Insofern existieren auch erstaunliche Parallelen zwischen Sekten wie Aum Shinrikyo, totalitären Weltanschauungs- und Heilsbewegungen wie Scientology, religiöspolitischen Bewegungen des rechten christlichen Spektrums und des gewaltbereiten Islamismus (Dschihadisten). ${ }^{15}$

\section{Die Ideologie des Islamismus und Dschihadismus}

Der Islamismus ist eine politische Ideologie, die sich einer religiösen Sprache bedient und beansprucht, die wahre Auslegung des Glaubens darzustellen. Er ist ein Gegenentwurf zu westlichen Ordnungs- und Wertvorstellungen. ${ }^{16}$ Der moderne Islamismus des 20. Jahrhunderts ist zugleich eine Protestbewegung gegen die eigenen, als tyrannisch empfundenen Regierungen, und den Einfluss des »Westens «, die für sozioökonomische Probleme, kulturelle Entfremdung und politische Ohnmacht der islamischen Welt verantwortlich gemacht werden. ${ }^{17}$ Rechtsgelehrte stellten sich die Frage nach der Ursache der »westlichen « Überlegenheit und des eigenen »Niedergangs «. Einige sahen als wichtigste Ursache die Tatsache an, dass sich der praktizierte traditionelle Islam weit von seinen Ursprüngen, den im Koran geforderten Lebensweisen, entfernt hatte. Die Schlussfolgerung lautete, einen Staat zu schaffen, in dem der Koran und die Überlieferungen des Propheten als Verfassung angesehen und das islamische Recht von den Gläubigen befolgt werden konnte. ${ }^{18}$

Vorläufer dieser Bewegungen traten zuerst Ende des 19. Jahrhunderts im Vorderen Orient auf. Der moderne Islamismus ist als eine »Zurück-zu-den-Wurzeln«-Bewegung in der Regel sehr konservativ in seiner Religionsinterpretation, die als einzig mögliche verstanden wird. Während sich Teile der islamistischen Bewegungen an demokratischen Wahlen beteiligen und sich an die rechtlichen Rahmenbedingungen ihrer Heimatländer halten, wollen andere die herrschenden muslimischen Regierungen mit Gewalt beseitigen und den »Westen« bekämpfen (Dschihadisten), um als Ziel einen islamischen Gottesstaat zu etablieren (Talibanisierung). ${ }^{19}$ Die Lagebeurteilung und das

14 Der Spiegel, 41/2001, S. 168-169 (»Krieg gegen Dämonen«).

15 Vgl. Hirschmann/Tophoven/Dietl (2006), Kap. 5.

16 Vgl. Innenministerium NRW (2004), S. 8.

17 Vgl. Steinberg (2002), S. 19.

18 Vgl. Heine (2001), S.88 ff.

19 Vgl. Innenministerium NRW (2004), S. 8. 
Ziel sind mithin bei Islamisten und Dschihadisten gleich. Ob der Weg dorthin notwendigerweise Gewalt mit einschließen muss, wird unterschiedlich beurteilt.

Die Ideologie für eine islamische Weltordnung und bewaffnete »Verteidigungsanstrengungen $\aleph^{20}$ (Dschihad) beruht vor allem auf den Arbeiten von zwei Gelehrten. ${ }^{21}$ Das neue islamistische Denken begann zwar in den 1920er Jahren in Ägypten und Indien, aber der Dschihadismus mit Terroranschlägen und Massenmord bekam seine heutige Ausrichtung und Qualität durch die grundlegenden Arbeiten des ägyptischen Lehrers und Theologen Sayyid Qutb (1906-1966), der ab den 1950er Jahren eine führende Rolle in der islamistischen ägyptischen »Muslimbruderschaft « einnahm. Qutb propagierte einen Kampf gegen den Westen, die Ablehnung seiner Werte und der kulturellen Moderne, die er in dem Buch »Ma'alim fi-1 Tariq « (Wegzeichen, Meilensteine) 1964 zusammenfasste. ${ }^{22}$ Es gilt noch heute als geistiges Fundament aller Mudschahiddin (Dschihad-Kämpfer). Sayyid Qutb wurde 1966 in Ägypten hingerichtet, seine Ideen von Mitstreitern und Anhängern wie Ahmad Jassin (HAMAS), Omar Abdel Rahman oder Mohammed Qutb (Bruder) aufgegriffen und in entsprechenden Zirkeln umgesetzt. Qutbs Theorien wurden konkretisiert und in Richtung der heutigen Dschihad-Interpretation verfeinert von Scheich Dr. Abdullah Azzam, einem Palästinenser, der in den 1970er und 1980er Jahren zum Vordenker des "globalen Dschihad « wurde. Zusammen mit Mohammed Qutb lehrte er in den 1970er Jahren an der Universität Dschidda in Saudi-Arabien. Er wurde akademischer und theologischer Lehrer Osama bin Ladens während dessen Studium in Dschidda.

Nach Sayyid Qutb befindet sich die (muslimische) Welt wieder im vorislamischen, heidnischen Zustand der »Dschahiliyya «. Den Grund für diesen allgemeinen Niedergang der Zivilisation sieht er darin, dass die Gesellschaften von heute ihre ganzheitliche religiöse Orientierung verloren hätten und lediglich an partikulare, menschliche Interessen gebunden seien. Das kann aber ein wahrer Muslim nach Sayyid Qutbs Ansicht nicht dulden und muss dagegen einschreiten, so wie der Prophet Mohammed gegen seine heidnischen Landsleute einschritt. Somit stünden die Muslime wieder am Scheideweg (mafraq attariq). Sie müssten dieselben Schritte befolgen wie seinerzeit der Prophet: die Bildung einer charismatischen Gruppe (dschama'a); den Auszug aus der heidnischen Umgebung (hidschra); den Kampf (dschihad), in dem sich die Gruppe der Gläubigen gegen die Gegner durchsetzt; und schließlich den Endzustand, der in der Wiedereinführung des Islam besteht. ${ }^{23}$ Eine derartige Bewegung müsse allerdings angestoßen werden durch tapfere Muslime, die vorangehen und eine Art »ausgewählte Elite« darstellen, die Sayyid Qutb als »Avantgarde« bezeichnet. Eine solche »Avantgarde « müsse die »Wiedererweckung « der islamischen Länder in Angriff nehmen, um die »Dschahiliyya « in einem Krieg zwischen dem »Guten« und dem »Bösen« von innen her zu zerstören.

20 Die Übersetzung »Heiliger Krieg« für den »Dschihad « ist falsch. Richtig ist vielmehr die Übersetzung "Anstrengung, Bemühung «.

21 Vgl. hierzu detailliert Hirschmann/Tophoven/Dietl (2006), Kapitel 5.

22 Der Titel wird unterschiedlich übersetzt, zum einen als »Meilensteine«, zum anderen als »Wegzeichen $«$.

23 Vgl. Rudolph (2000), S. 101.
Sayyid Qutbs ideologische Grundlagen wurden nach seiner Hinrichtung in Ägypten 1966 von zahlreichen Gelehrten der Muslimbruderschaft in Richtung "gewaltsamer Dschihad « fortentwickelt und verfeinert. Einer der wichtigsten unter ihnen war Scheich Dr. Abdullah Azzam (1941-1989), ein Ideologe aus Palästina, der u.a. das logistische Netzwerk aufbaute, um Geld und Freiwillige in den Kampf gegen die sowjetischen Truppen in Afghanistan zu schleusen. Seine radikal internationale Vision des »Dschihad « übte großen Einfluss auf viele Mudschahiddin-Kämpfer aus. ${ }^{24}$ »Jedes Prinzip braucht eine Vorhut, die es voranträgt und schwierige Aufgaben und ungeheuer große Opfer auf sich nimmt. Es gibt keine Ideologie, weder eine irdische noch eine himmlische, die keine Avantgarde braucht, die alles, was sie besitzt, für den Sieg einsetzt. (...) Diese Vorhut bildet das starke Fundament (al qaeda alsul-bah) für die Gesellschaft, auf die wir warten. « Und: »Eine kleinere Gruppe innerhalb dieser Schar bilden diejenigen, die aus dem weltlichen Leben fliehen, um ihr Handeln auf diese Ziele auszurichten und andere für sie zu gewinnen. Eine noch kleinere Gruppe innerhalb dieser Elite sind diejenigen, die ihre Seele und ihr Blut darangeben, um den Zielen und Prinzipien zum Sieg zu verhelfen «, so lauteten zwei der Lehren von Azzam. ${ }^{25}$ Dabei bildet das zitierte »starke Fundament « (al qaeda al-sul-bah) sowie die kleine Elite, die ihr Blut gibt, die Grundlage für die Namensgebung und das Selbstverständnis der späteren »Al-Qaida «, die sich als eine derartige Vorhut und Avantgarde begreift. Azzam wurde 1989 durch eine Autobombe in Pakistan getötet.

Die ideologischen Lehren und Weiterentwicklungen führten zu einem seit den 1980er Jahren mit wenigen Variationen weltweit gültigen »3-2-1-Modell«, in dem von drei Feinden, zwei Angriffsarten und einer notwendigen Gegenmaßnahme, dem Dschihad, ausgegangen wird ${ }^{26}$ :

- Drei Feinde: Der Ausgangspunkt des Modells ist der Kern der islamistischen Botschaft, dass die Fehlentwicklungen in den muslimischen Gesellschaften ursächlich darauf zurückzuführen seien, dass Muslime im Laufe der Zeit von ihren Feinden immer weiter vom »eigentlichen Glauben « entfremdet wurden, was es zu korrigieren gelte. Dazu seien die Muslime jedoch zunächst nicht in der Lage, da ihre Feinde eine Rückbesinnung, Erstarkung und Wohlstand verhindern würden. Als »Feinde « verstanden werden: »Kreuzfahrer « (westlichchristliche Gesellschaften inklusive Russland), »Juden « (der Staat Israel) und »Handlanger« (eigene muslimische Regierungen als devote Erfüllungsgehilfen der »Ungläubigen«). Deren Einfluss müsse zunächst zurückgedrängt werden.

- Zwei Angriffsarten: Da die Ungläubigen und Handlanger das Feld nicht freiwillig räumen würden, sei Gewalt in einem »Dschihad « der einzig erfolgversprechenden Weg. Dabei missbrauchen sie die »Dschihad-Lehren « des Islam, denen zufolge ein »Dschihad « als kollektive Verteidigungsanstrengung aller »Rechtgläubigen « ausgerufen werden kann, wenn Muslime unterdrückt und vertrieben oder ihr Land okkupiert wird. »Dschihadisten« sehen die Muslime dauerhaft angegriffen und in ihrer Existenz bedroht, wogegen eine ge-

24 Vgl. Burke (2005), S. 353.

25 Zitiert in Burke (2005), S. 26 und 67.

26 Siehe hierzu detailliert Hirschmann/Tophoven/Dietl (2006), Kapitel 5 und 6. 
meinsame Verteidigungsanstrengung zwingend erforderlich sei. Dieser Angriff erfolge auf zwei Arten: zum einen durch die Eroberung und Okkupation muslimischen Landes (physischer Angriff), dem eine Unterdrückung der Menschen und Ausbeutung der Reichtümer und Ressourcen der Region folge; zum anderen durch den aggressiven Export von westlichen Werten in die muslimische Welt z.B. durch Tourismus, multinationale Konzerne, Globalisierung und Entwicklungszusammenarbeit (psychischer Angriff).

- Eine gemeinsame Verteidigungsanstrengung: Gegen die Feinde und ihre ständigen Angriffe sei zur Verteidigung ein »Dschihad « erforderlich. Zum Kampf des »Dschihad « werden nun, insbesondere in den Konfliktregionen, freiwillige Kämpfer aus dem gesamten Bereich der muslimischen Gemeinde (Umma) zusammengeführt, um als »dschihadistische Internationale« ihrer vermeintlich religiösen Pflicht nachzukommen. Diese Kämpfer aus allen Ländern und Regionen, in denen Muslime leben, werden als »Mudschahiddin« (Gläubige, die den Dschihad kämpfen) bezeichnet. Der Kampf fand bisher in der Praxis »zweigleisig « statt. Zum einen strömten in den Konfliktregionen als Streitmacht gegen die behaupteten Besetzungen fanatisierte Kämpfer zusammen, die überall in muslimischen Gemeinden rekrutiert worden waren. Solche »lokalen Dschihads« als Terrorkampf fanden und finden z.B. statt in Afghanistan (1980-1988, »Al-Qaida «), Palästina (seit 1987, HAMAS und die Gruppe »Islamischer Dschihad «), Tschetschenien (seit 1998; Mudschahiddin unter Shamil Bassajew), Bosnien-Herzegowina (1992-1995, »Al-Qaida«), Irak (seit 2003, Mudschahiddin unter Abu Mussab al-Sarkawi bis Juni 2006, seither unter seinem Nachfolger Abu Hamza al-Muhadschir) und in Südostasien (seit Mitte der 1990er Jahre, Jeemaah Islamiya). Zum anderen finden Terroranschläge als »Nadelstichtaktik im Herzen der Feinde« mit dem Ziel der Politikbeeinflussung statt wie z.B. in New York und Washington (11.09.2001), auf Djerba/Tunesien gegen deutsche Touristen (11.04.2002), auf Bali/Indonesien gegen Touristen (12.10.2002), in Istanbul (15. bzw. 20.11.2003), Madrid (11.04.2004) und London (07. bzw. 21.07.2005).

Diese Weltsicht und Ideologie bestimmt den gewaltsamen »Dschihad-Terrorismus « seit den 1980er Jahren bis heute und auf absehbare Zeit auch in der Zukunft. Damit erklärt sich auch das Selbstbewusstsein eines Osama bin Laden, der selbst ein »Angeworbener « für diese Ideologie ist, als er kurz vor dem 11.09.2001 einem pakistanischen Journalisten erklärte: »Jeder von uns kann mehrfach ersetzt werden.« Diese Behauptung wurde seither auch immer wieder, zuletzt bei der Nachfolge von Abu Mussab al-Sarkawi im Juni 2006, eindrucksvoll unter Beweis gestellt. Entscheidend sind die Ideen in den Köpfen und die Radikalisierung von Sympathisanten, nicht aber einzelne Personen. Hierarchische Führer im traditionellen Sinne existieren nicht; Osama bin Laden zum Beispiel bezieht seine Stellung und Reputation aus seiner Management-Fähigkeit und seinem fortgesetzten Engagement für die Anwendung der Ideologie. Es wäre aber ohne weiteres denkbar, dass sich im Falle seines Todes binnen kürzester Zeit im »Dschihad-Netzwerk« Personen mit vergleichbaren Fähigkeiten und ähnlicher Leidenschaft finden ließen.
Personen wie Osama bin Laden, Ayman al-Sawahiri oder Abu Mussab al-Sarkawi können daher eher als »Propagandisten des Dschihad« bezeichnet werden. Die von ihnen geleiteten Organisationen erbringen als eine Art »Provider « Dienstleistungen für die »Dschihad-Kämpfer « (Mudschahiddin). Derartige Provider-Leistungen umfassen z.B. die Bereitstellung bzw. Schaffung von Ausbildung, Waffen, Logistik, Koordinierung, Organisation, ideologischem Rat, Netzwerkstrukturen und Finanzmitteln. Bei Ideologien, die in sektenartigen Strukturen organisiert werden, läuft aber die traditionelle personenbezogene Terrorismusbekämpfung ebenso ins Leere wie die Anwendung klassischer Machtinstrumente. Eine Gewaltidee verliert nicht an Einfluss und Popularität, wenn einzelne ihrer Propagandisten getötet werden oder wenn gegen ihre Anhänger Gewalt ausgeübt wird. Im Gegenteil wird sie sogar an Zuspruch gewinnen, wie die Situation im Irak seit 2003 gezeigt hat.

\section{Sektenausrichtung und Bekämpfung}

Unter Anwendung der zuvor dargestellten Kriterien ist der gewaltbereite Islamismus bzw. Dschihadismus eindeutig als Sekte klassifizierbar:

- Er vertritt eine in sich geschlossene, von der überwiegenden Mehrheit abweichende Lehre und steht als weltanschaulichpolitische Bewegung in Opposition zu seiner Umgebung, deren Ausrichtung und Werte er zurückweist. Es handelt sich ohne Zweifel auch um eine ideologische Gemeinschaft mit gewaltbefürwortender Lehre.

- Markant ist die Aufteilung der Welt in »Rechtgläubige« und »ungläubige Feinde« (»Dualismus«). Die gewaltbereiten Dschihadisten bilden nach Selbsteinschätzung eine »Elite« bzw. »Vorhut «, die wegen ihrer Verbreitung der Wahrheit von den drei Feinden verfolgt wird (»verfolgte Auserwählte $«$ ). Da sie aus eigener Sicht ausschließlich den Willen Allahs umsetzen, sind ihre Ziele nicht verhandelbar, ihr Kampf gerechtfertigt und der Sieg gewiss (»Determinismus«). Ihren wahren Glauben beweisen und Märtyrer werden können nur solche Mudschahiddin, die am Kampf teilnehmen bzw. in ihm getötet werden (»Rettung durch Konflikt«). Die Werte und Regeln des Gegners sind keine zu respektierenden Normen und eine Vielzahl von Opfern ist nicht das Problem, sondern ein Teil von dessen Lösung (fehlende Hemmschwellen).

- Die totalitäre Struktur ergibt sich aus der Radikalität und Exklusivität der Lehre. Die Führerfiguren des Dschihadismus geben ideologische Leitlinien und Verhaltensanweisungen heraus, die verbindlich sind. Abweichungen davon werden nicht toleriert.

Die Stärke jedes Fundamentalismus liegt in einem Autoritarismus, der seine Moral auf etwas gründet, was als absolute Wahrheit gilt. ${ }^{27}$ Die »Dschihadisten « sind dabei bereit, für ihr nach dieser »Wahrheit « zurechtgebogenes Realitätsempfinden zu sterben und zu morden. ${ }^{28}$ Aufbau, Ideologie, Strukturen

27 Kramer/Alstad (1995), S. 222.

$28 \mathrm{Zu}$ der Frage, wie Menschen dazu gebracht werden, sich einer Sekte bzw. einem Guru zu unterwerfen, vgl. detailliert Kramer/Alstad (1995). 
und Interpretation der politisch-gesellschaftlichen Situation lassen im Ergebnis die Bezeichnung »Sekte« zu. Die Organisationsform und das Weltbild des dschihadistischen Terrorismus als Gewaltidee zeigen, dass es sich nicht um eine hierachischhomogene Terrorgruppe klassischer Prägung handelt. Durch den Plattform-Charakter und die multinationale Ausrichtung kann zudem das Aktionsspektrum regional nicht mehr eingegrenzt werden. Eine Ideologie reicht überall dort hin, wo sich Anhänger dafür werben lassen. Dennoch darf nicht übersehen werden, dass es sich um eine absolute Minderheitenströmung handelt, die den Islam mit einer eigenen politischen Interpretation missbraucht. Unter Muslimen finden sich heute ca. fünf bis sieben Prozent Islamisten, von denen wiederum nur ca. zehn Prozent für dschihadistische Ideen aufgeschlossen sind. Aus diesem Potenzial werden die Radikalsten als Mudschahiddin rekrutiert.

Die entscheidende Frage bei der Bekämpfung des islamistischen Terrorismus muss mithin lauten: Wie kann eine Gewaltideologie in den Köpfen bekämpft werden, die von einer sektenartigen Minderheit in Umlauf gebracht und beworben wird?

Eine Ideologie kann international nicht erfolgreich mit klassischen Machtinstrumenten wie z.B. Streitkräften, Polizei und repressiver Diplomatie bekämpft werden. Dies gilt schon deshalb, weil eine Selektion zwischen der gewaltbereiten Minderheit und der friedlichen Mehrheit der Bevölkerung im Alltag und mit dem Wissen der Bekämpfer kaum gelingen kann. Eine »Politik der harten Hand « wie z.B. in Tschetschenien oder im Irak kann daher nicht zielgenau ausfallen, sondern trifft vor allem Nicht-Kombattanten. Repressionen und Bekämpfungsaktionen gefährden in der Hauptsache das Leben und den Besitz derjenigen, die nicht Anhänger dschihadistischer Gewaltideen sind, weil den Bekämpfern eine exakte Unterscheidung auch auf Grund der Taktik der Gewalttäter als »Fische im Wasser « der Bevölkerung bis zur Unmöglichkeit erschwert wird. Das nutzen die »Dschihadisten « wiederum für ihre Propaganda, zur Verschleierung des Urhebers der Gewalt und zur Rechtfertigung für weitere Gewaltaktionen, indem sie das Motto »Die Ungläubigen kämpfen gegen alle Muslime und unterdrücken sie« mit den Ergebnissen der fehlenden Treffgenauigkeit des Gegners unterfüttern. Hiermit ziehen sie wiederum neue Sympathisanten als »Betroffene der Terrorismusbekämpfung « auf ihre Seite. Daher ist vom Einsatz klassischer Machtinstrumente grundsätzlich abzuraten, da sie eher als Brandbeschleuniger denn als Löschwasser wirken.

Ebenso wenig ist eine personenbezogene Bekämpfung erfolgversprechend. Es wirkt beinahe hilflos, einige bekannte Dschihadisten auf Steckbriefen zu veröffentlichen und hohe Summen auf ihre Ergreifung (tot oder lebendig) auszuloben. Die Ideologie wird dadurch nicht getroffen. Zwar ist es angezeigt, bekannte Dschihad-Fanatiker aller Ebenen international zu ergreifen und als pathologisch Gewaltbereite einer Strafverfolgung zuzuführen, aber es muss als »zweite Schiene« der Bekämpfung vor allem sichergestellt werden, dass die Propagandisten der Ideologie nicht stetig neue Sympathisanten in hoher Zahl rekrutieren können. Dies funktioniert nur über gesellschaftspolitische Ansätze, sprich: eine theologische und politische Auseinandersetzung mit der Ideologie. ${ }^{29}$

\section{Einige Beispiele:}

Hierzu gehört zunächst, den Islamisten nicht mehr das Feld der privaten Sozialleistungen und der Milderung von Armut zu überlassen. Islamisten sind besonders bei denjenigen erfolgreich, die die wirtschaftlichen, sozialen oder kulturellen Probleme ihres Alltags nicht allein bewältigen können. Sie stellen wirtschaftliche Gerechtigkeit in Aussicht, lindern soziale Missstände durch die Bereitstellung von Dienstleistungen und verschaffen Selbstbewusstsein und Halt durch aktive Mitarbeit. ${ }^{30}$ Damit erhalten sie auch für ihre radikalen Ansichten ein »dankbares« Auditorium. Gemäßigte Organisationen vor Ort könnten hierzu mit finanziellen Mitteln ausgestattet werden, denn materielle Not und individuelle Perspektivlosigkeit waren stets Wasser auf die Mühlen der Demagogen mit den »einfachen Lösungen $«$.

Eine religiös-politische Auseinandersetzung mit der Gewaltideologie des »Dschihadismus « muss gesellschaftlich aber vor allem aus den muslimischen Gemeinden heraus geleistet werden. Die »Lehren« der Dschihadisten müssen immer wieder öffentlich als mit dem Glauben unvereinbare Handlungen aufgegriffen und wiederlegt werden. Dies gilt besonders in Moscheen, Kulturvereinen und anderen muslimischen Einrichtungen. Bedauerlicherweise ist es so, dass bisher nur fundamentalistische Quellen über die finanziellen Mittel verfügen, in Büchern, Broschüren und Videos »den Islam« zu erläutern. Benötigt wird eine internationale muslimische Bewegung, die islamistische Umtriebe in ihren jeweiligen Gemeinden konsequent zu unterbinden versucht. Hierzu bedarf es auch der finanziellen und politischen Unterstützung, um Aufklärungsarbeit leisten und ein entsprechendes Problembewusstsein bei den Muslimen schaffen zu können. Von entscheidender Bedeutung ist dabei auch, den Reformislam in den muslimischen Staaten zu stärken, der sich fatalerweise überall in der Opposition befindet und von zwei Seiten angegriffen wird: zum einen von den Islamisten und Dschihadisten, die ihn als unislamisch denunzieren, zum anderen durch die eigenen despotischen Regierungen, die an Reformen, Modernisierung und Pluralismus überhaupt kein Interesse haben. Diese »SandwichPosition« nimmt dem Reformislam häufig die Luft zum Atmen, denn entgegen der populären Huntington-These des »Kampfes der Kulturen « findet die eigentliche Auseinandersetzung nicht zwischen Christentum und Islam, sondern innerhalb des Islam zwischen Reformkräften und Islamisten statt. ${ }^{31}$

\section{Literatur- und Quellenverzeichnis}

Abercrombie, N./Hill, St./Turner, B. S. (1994): Dictionary of Sociology, London.

Beckers, H.-J./Kohle, H. (1994): Kulte, Sekten, Religionen - Von Astrologie bis Zeugen Jehovas, Augsburg.

29 Siehe hierzu auch Hirschmann (2005), Punkt 3.

30 Vgl. Steinberg (2002), S. 28.

31 Hirschmann (2005), Punkt 3. 
Bergen, P.L. (2001): Heiliger Krieg Inc. - Osama Bin Ladens Terrornetz, Berlin.

Bierbrauer, G. (2002): Einige Anmerkungen zu den Ursachen des Internationalen Islamischen Terrorismus; in: Hanns-Seidel-Stiftung, Politische Studien, Heft 386, Nov./Dez. 2002, S. 61-64.

BMFS (2003): Prävention im Bereich der »Sogenannten Sekten und Psychogruppen ", Abschlussbericht der wissenschaftlichen Begleitung zum Modellprojekt des Bundesministeriums für Familie, Senioren, Frauen und Jugend.

Bundesministerium des Innern, Hrsg. (2004): Islamismus, Berlin.

Burke, J. (2005): Al-Qaida - Wurzeln, Geschichte, Organisation, Düsseldorf und Zürich.

Cammans, H.-M. (1998): Sekten - Die neuen Heilsbringer? Düsseldorf.

Canadian Security Intelligence Service (2000): Doomsday Religious Movements, CSIS-Report 2000/03, 18.12.1999.

Clarke, R.A. (2004): Against all Enemies, München.

Enquete-Kommission (1998): Endbericht der Enquete-Kommission "Sogenannte Sekten und Psychogruppen ", Deutscher Bundestag, 13. Wahlperiode, Drucksache 13/10950, Bonn.

Frank, H./Hirschmann, K, Hrsg. (2002): Die weltweite GefahrTerrorismus als internationale Herausforderung, Berlin.

Gasper, H./Müller, J./ Valentin, Fr., Hrsg. (1990): Lexikon der Sekten, Sondergruppen und Weltanschauungen, Fakten, Hintergründe, Klärungen, Freiburg i.Br.

Glagow, R. (2001): Die Dschihad-Tradition im Islam; in: MeierWalser, R.C./ Glagow, R., Hrsg. (2001): Die islamische Herausforderung, Aktuelle Analysen 26, Hanns-Seidel-Stiftung, München, S. 37-66.

Heine, P. (2001): Terror in Allahs Namen - Extremistische Kräfte im Islam, Freiburg i.Br.

Hirschmann, K. (2003): Terrorismus, Hamburg.

Hirschmann, K./Leggemann, Chr., Hrsg. (2003): Der Kampf gegen den Terrorismus - Strategien und Handlungserfordernisse, Berlin.

Hirschmann, K. (2005): Der Dschihadismus: Ideologie, Organisation und Bekämpfungsmöglichkeiten, Beitrag zum Themenbereich »Terrorismus - Eine neue Form der Kriegsführung ", BerlinBrandenburgische Akademie der Wissenschaften (i.E.).

Hirschmann, K./Tophoven, R./Dietl, W. (2006): Das Lexikon des Terrorismus - Täter, Opfer, Hintergründe, Frankfurt a.M.

Innenministerium NRW (2004): Islamismus - Instrumentalisierung der Religion für politische Zwecke, Düsseldorf.
Johnson, B. (1971): Church and Sect Revisited; in: Journal for the Scientific Study of Religion, Bd. 10, 1971.

Juergensmeyer, M. (2004): Terror im Namen Gottes, München.

Kepel, G. (2002): Das Schwarzbuch des Dschihad - Aufstieg und Niedergang des Islamismus, München.

Kramer, J./ Alstad, D. (1995): Masken der Macht - Warum Menschen bereit sind, sich einem Guru zu unterwerfen, Frankfurt a.M.

Mandau, L. (1995), Tödlicher Sektenwahn - Die teuflischen Tricks der Seelenfänger, München.

Rashid, A. (2001): Taliban - Afghanistans Gotteskrieger und der Dschihad, München.

Reller, H./Kießig, M./Tschoerner, H. (1978): Handbuch Religiöse Gemeinschaften - Freikirchen, Sondergemeinschaften, Sekten, Weltanschauungen, Missionierende Religionen des Ostens, Neureligionen, Psycho-Organisationen, Gütersloh.

Reuter, Chr. (2002): Mein Leben ist eine Waffe - Selbstmordattentäter: Psychogramm eines Phänomens, München.

Rohmann, D. (1998): Mögliche Prädisposition einer Sekten- und Kultmitgliedschaft, Diplomarbeit eingereicht an der Philosophisch-Pädagogischen Fakultät der Katholischen Universität Eichstätt (im Internet veröffentlicht).

Rudolph, U. (2000): Überlegungen zum radikalen Islamismus - Die Lehren des Sayyid Qutb; in: Neue Zürcher Zeitung, Nr. 117, 20.05.2000, S. 101.

Schneckener, U. (2002): Netzwerke des Terrors: Charakter und Strukturen des Terrorismus, SWP-Studie 42/02, Berlin.

Senatsverwaltung Berlin (1997): Risiken und Nebenwirkungen: Informationen zu ausgewählten religiösen und weltanschaulichen Bewegungen und Psychoangeboten, Berlin.

Stark, R./Bainbridge,W. S. (1985): The Future of Religion, Berkeley.

Steinberg, G. (2002): Islamismus und islamistischer Terrorismus im Nahen und Mittleren Osten - Ursachen der Anschläge vom 11. September 2001, Broschürenreihe »Zukunftsforum Politik« Nr. 39, Konrad-Adenauer-Stiftung, Bonn.

Van Der Straeten, St. (2003): Im Bann der Psychosekten - Eine Analyse zum Phänomen mit Präventionsmöglichkeiten, Wissenschaftliche Hausarbeit, Pädagogische Hochschule Heidelberg (im Internet veröffentlicht).

Wilson, B. R. (1970): Religiöse Sekten, München.

Zimbardo, P.G. (1997): Jeder kann von einer Sekte verführt werden; in: Psychologie Heute, 11, S. 34-37. 